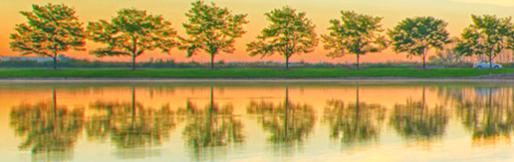

\section{Physics Models in Detector Simulation}

\section{Daniel Elvira} HSF Workshop - how 2019 March 20th, 2019
This manuscript has been authored by Fermi Research Alliance, LLC under Contract No. DE-AC02-07CH11359 with the U.S. Department of Energy, Office of Science, Office of High Energy Physics. 


\section{Scope and goals, Outline}

Overview of the detector simulation physics models needs of HEP experiments

- Restricted to LHC experiments (ALICE, ATLAS, CMS, LHCb) and Belle II

- Other experiments are discussed in other talks

- Focused on the Geant4 toolkit

Discuss differences and commonalities among experiments

Identify opportunities for collaboration

- Introduction

- Physics in Geant4, challenges identified during the HSF roadmap and Simulation CWP process

- Reports from the experiments - recent developments, tests, needs

- Summary and outlook 


\section{Introduction: physics in Geant4}

\section{Very few of our HEP colleagues know how physics is handled within Geant4}

- Tens of models to describe different EM, hadronic, decay processes (sub-eV to TeV)

- E.g. of EM: Compton, Photoelectric, ionization, bremsstrahlung, multiple scattering, ionization

- E.g. of HAD: stopping, decay, elastic and inelastic models, capture models, fission

- Theory-based and parametric models

- Theory-based preferred for prediction power in regions with little or no data

- The user builds a modular "Physics List" from individual models for different particles and energy ranges, including transition regions of phase space

- Ready-to-use physics lists: e.g. QGSP_BERT, FTFP_BERT, QGSP_INCLXX

- QGSP, BERT, INCL refer to different theory-based models for hadron-nucleus interactions

- Thin-target data used by G4 Collaboration to develop and initially tune physics models

- Test beam (TB) and collider data is used for validation and small tweaks to physics lists

- Sometimes a better thin target tuning does not improve physics for TB or collider data 


\section{Introduction: the HSF roadmap and simulation CWP}

Physics challenge: review G4 models, including assumptions, approximations, limitations for higher precision and faster runtime through software modernization

- EM shower shapes, spatial resolution - large impact on $H \rightarrow \gamma \gamma$ event modeling, lepton ID and isolation at LHC

- Improvements to multiple scattering, pair production and bremsstrahlung in 2010-2015
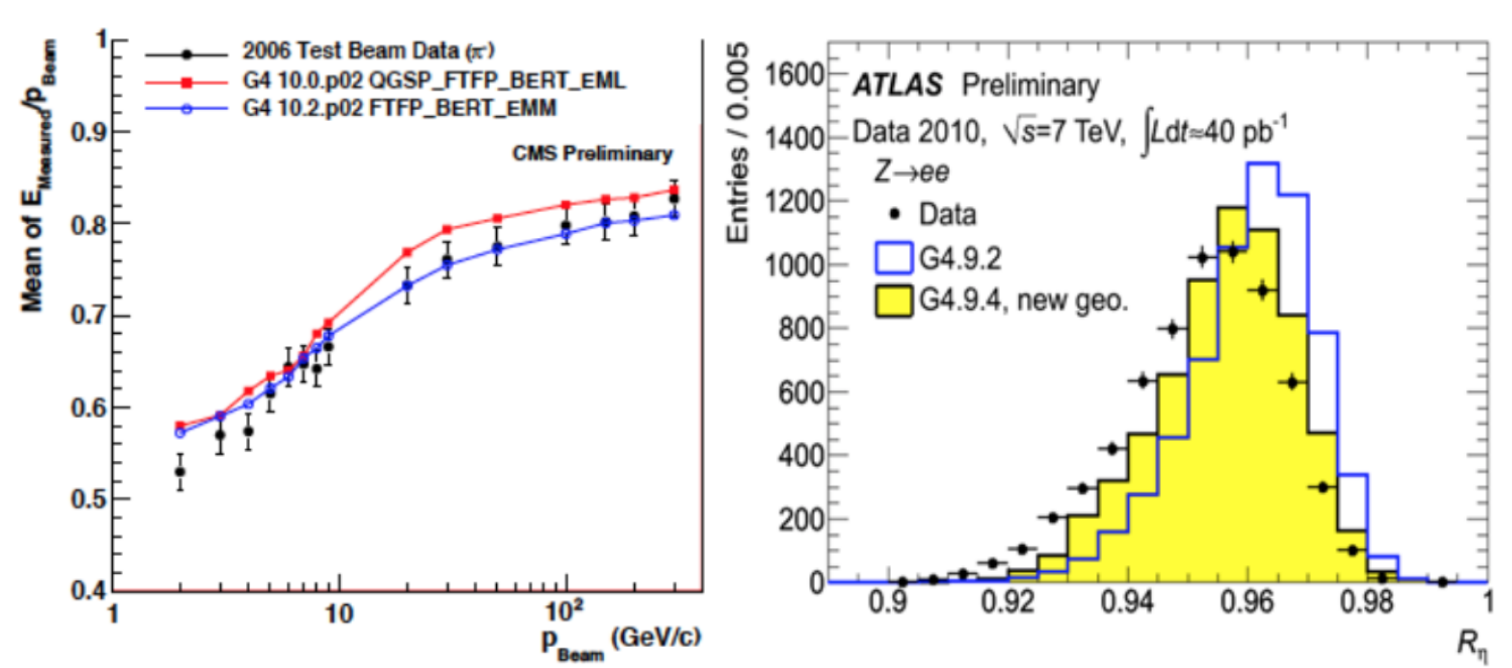

CMS $\pi^{-}$and $\pi^{+}$response (TB 2006) Note significant improvement in G4-todata agreement when moving from the EML (simplified) to the EMM (detailed) multiple scattering model

ATLAS $\mathbf{R}_{\eta}$ for electrons (2010 data) Ratio of energy in $3 \times 7$ and $7 \times 7 \eta-\phi$ cells Shower narrower in simulation, most likely due deficiencies in EM models 


\section{Introduction: the HSF roadmap and simulation CWP}

Physics challenge: review G4 models, including assumptions, approximations, limitations for higher precision and faster runtime through software modernization

- HAD shower shapes, energy response function - large impact on LHC hadronic final states, missing transverse energy, energy leakage beyond calorimeter limits

- Hadronic sequence: HE hadron-nucleon collision (parton string model), propagation within nucleus (intra-nuclear cascade model), de-excitation and evaporation (pre-compound, breakup)

- Parton string models: quark-gluon string (QGS), Fritiof (FTF); intra-nuclear cascade models: Bertini, binary, INCL++

- Most used physics list in HEP is FTFP_BERT (Bertini: 0-12 GeV, FTF: > 3 GeV since G4 10.3)

- Intra-nuclear and de-excitation at low and intermediate energy is key for modeling lateral shower shapes. Quasi-elastic and diffraction at HE defines longitudinal shapes

- HAD energy response accurate to a few \% and resolution to $10-20 \%$

- CALICE data suggests that G4 shower lateral profiles too narrow 


\section{Detector simulation physics: CMS}
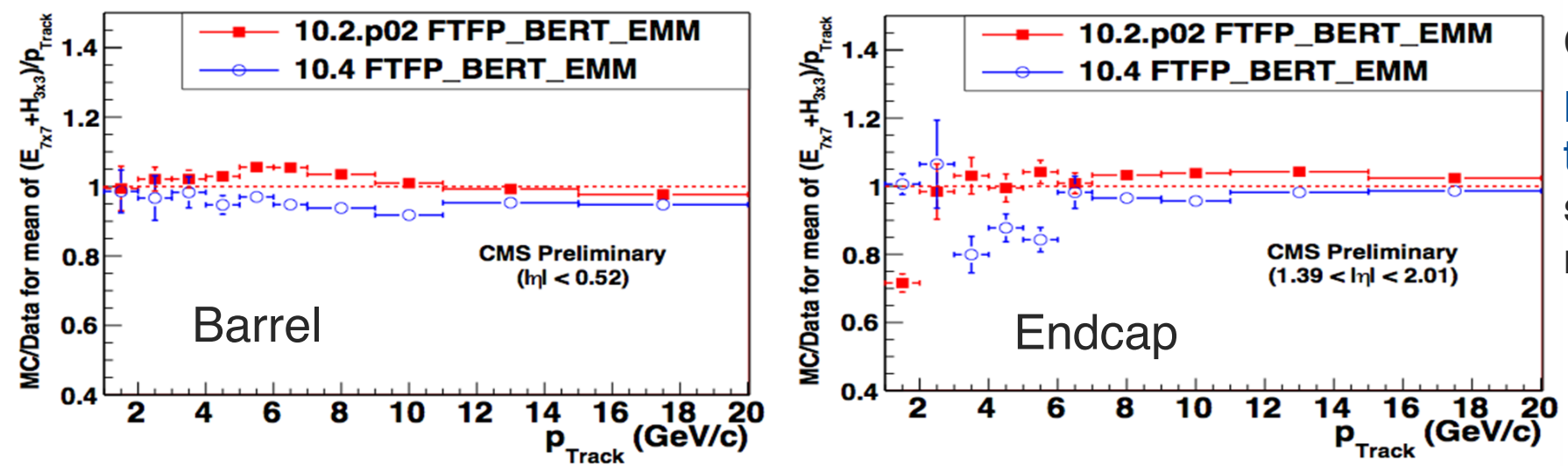

Collider data

MC-to-data ratio of tracker-to-calorimeter single track energy response ratio Agreement within 5-10\% in range with high stats.
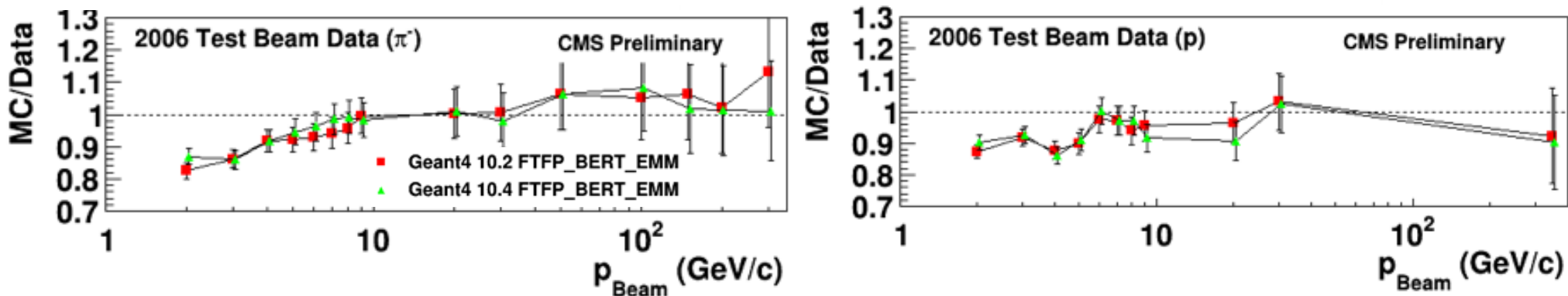

Test Beam 2006 MC-to-data ratio of $\pi$ and $p$ energy resolution

Good agreement between G4 prediction and data for $p_{\text {Beam }}>6 \mathrm{GeV}$ within uncertainties Deviation of G4 prediction from data increases below $10 \mathrm{GeV}$ to up to $~ 15 \%$ at $2 \mathrm{GeV}$ 


\section{Detector simulation physics: CMS}

- High Granularity Calorimeter (HGCAL) to replace existing endcap calorimeters

- Unprecedented transv. and long. segmentation for EM (Si) and HAD (scintillator) sub-systems

- Enhanced PFlow calorimetry, pileup rejection, and particle ID. Measure shower fine structure
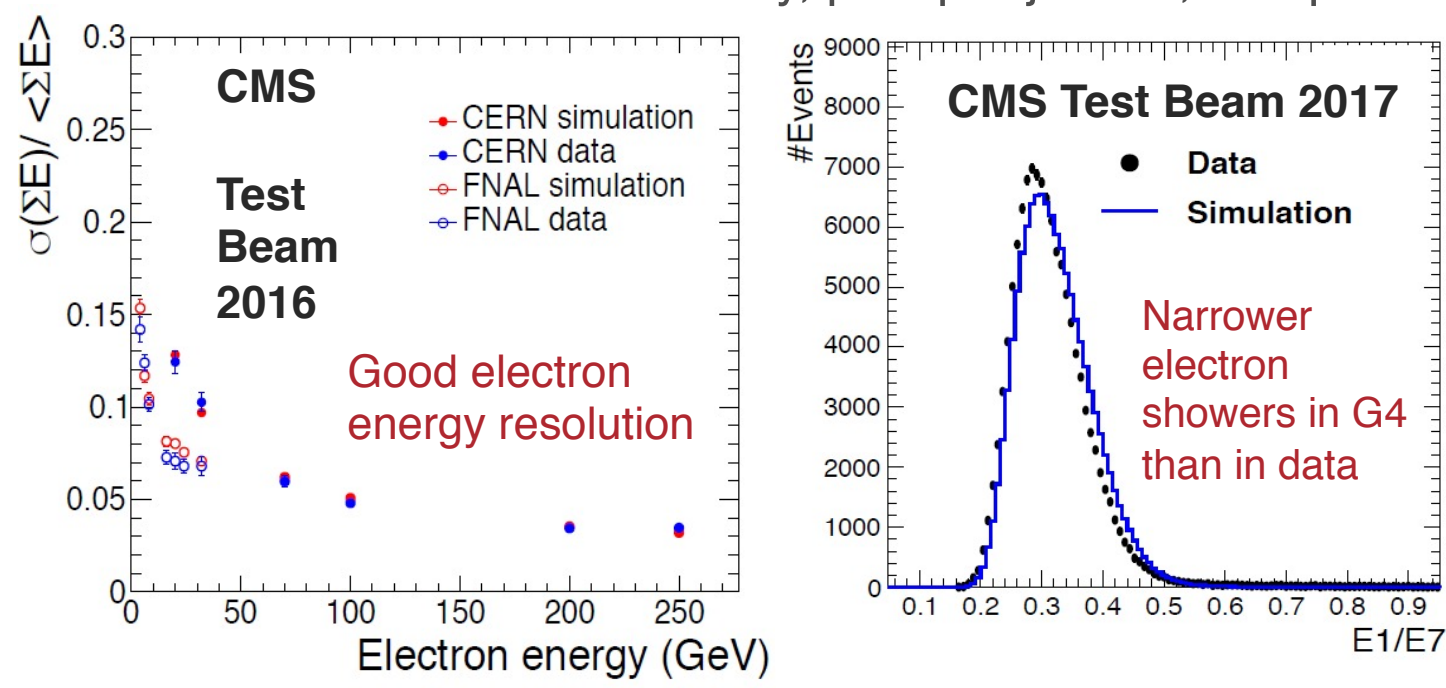

The more precise _EMY and EMZ physics lists introduce large CPU penalties for HL-LHC configurations including HGCAL

\section{Geant4 10.2 FTFP_BERT_EMM}

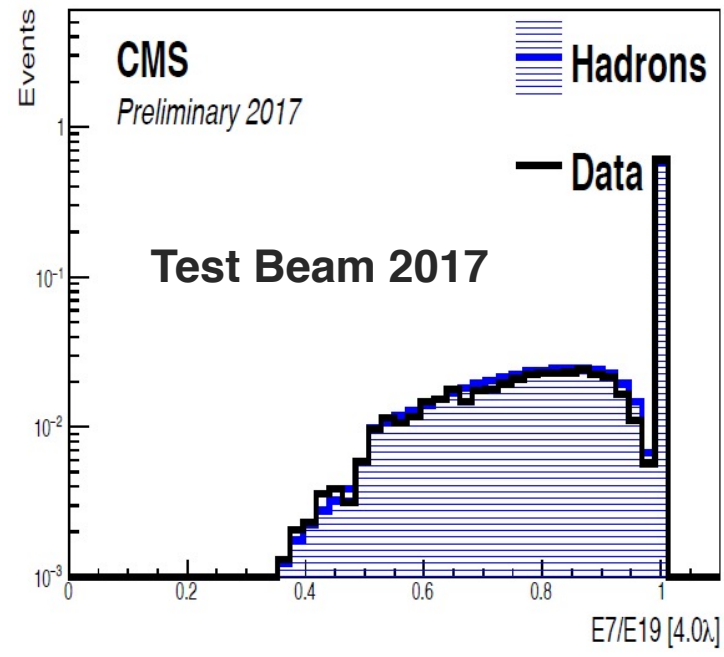

军 Fermilab 


\section{Detector simulation physics: ATLAS}

Multi-purpose experiment at the LHC collider focused on characterization of the Higgs boson and searches for beyond the Standard Model physics including dark matter

Recently, ATLAS focused on tuning Russian Roulette (NRR) approximation and secondary particles production cuts in range for EM processes - improve time performance

- Tune NRR algorithm parameters: energy threshold (E) and weight (w)

- New neutron tracks with $E_{k i n}<E$ are terminated with a probability of (w-1)/w

- Remaining neutrons below $E$ and their secondaries are weighted by w, i.e. their energy deposits in calorimeters are multiplied by $\mathrm{w}$

- Modify Geant4 default cuts in range for conversion, photoelectric, Compton processes

For all studies, ATLAS used G4 10.01.patch03 (plus additional patch - atlas07: geometry fixes and G4NystromRK4 stepper)

Setup uses shower libraries in FCal for e, $\gamma$, and neutrons and fast simulation in forward parts of the beam pipe (shielding blocks beyond the sensitive detectors). No pileup 


\section{Detector simulation physics: ATLAS}

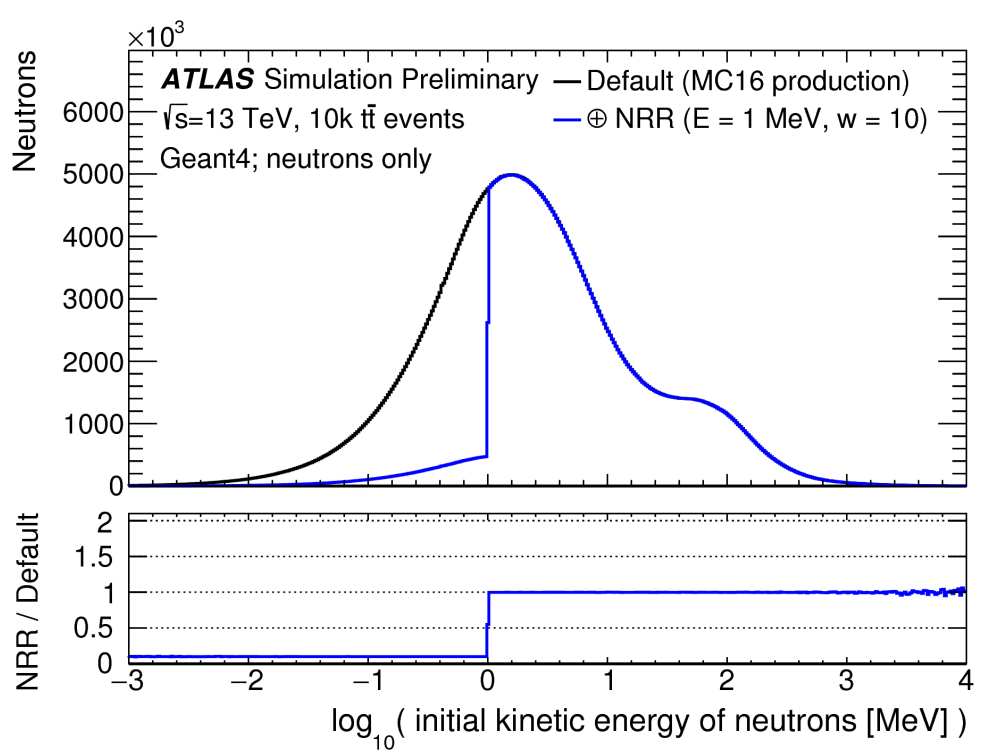

Initial kinetic energy of neutrons

The black curve corresponds to default setup and the blue curve shows the distribution for the default setup plus the Neutron Russian roulette (NRR) algorithm with energy threshold $1 \mathrm{MeV}$ and weight 10
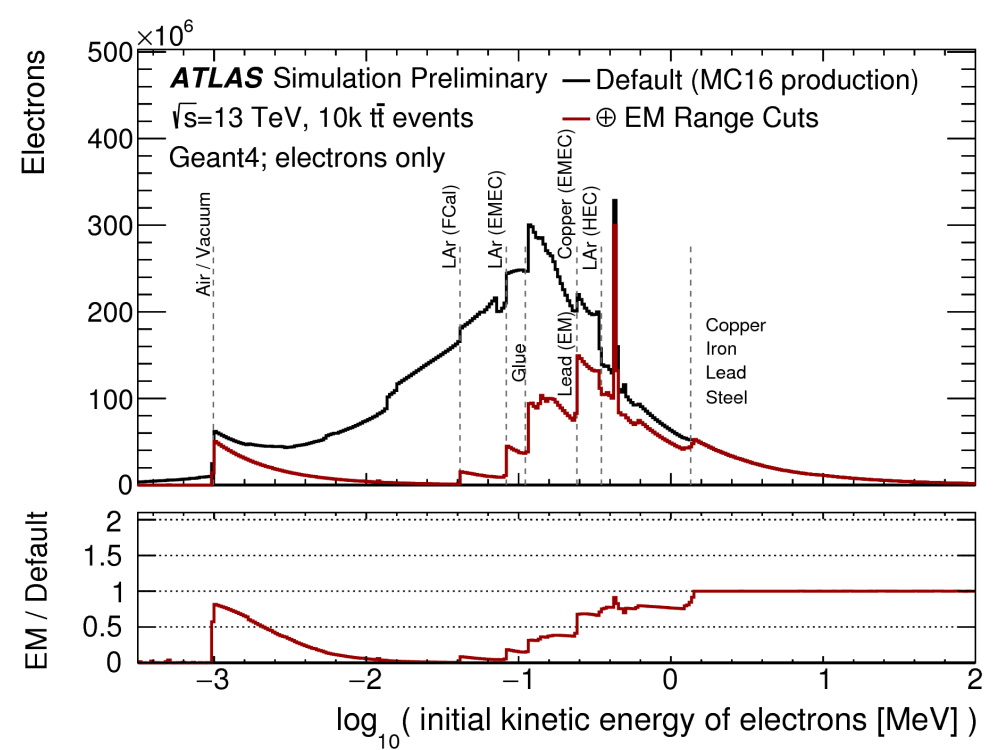

\section{Initial kinetic energy of electrons}

The black curve corresponds to default setup and the red curve shows the distribution for the default setup plus the added range cuts for electromagnetic processes. Vertical lines indicate cuts for some typical detector materials 


\section{Detector simulation physics: ATLAS}

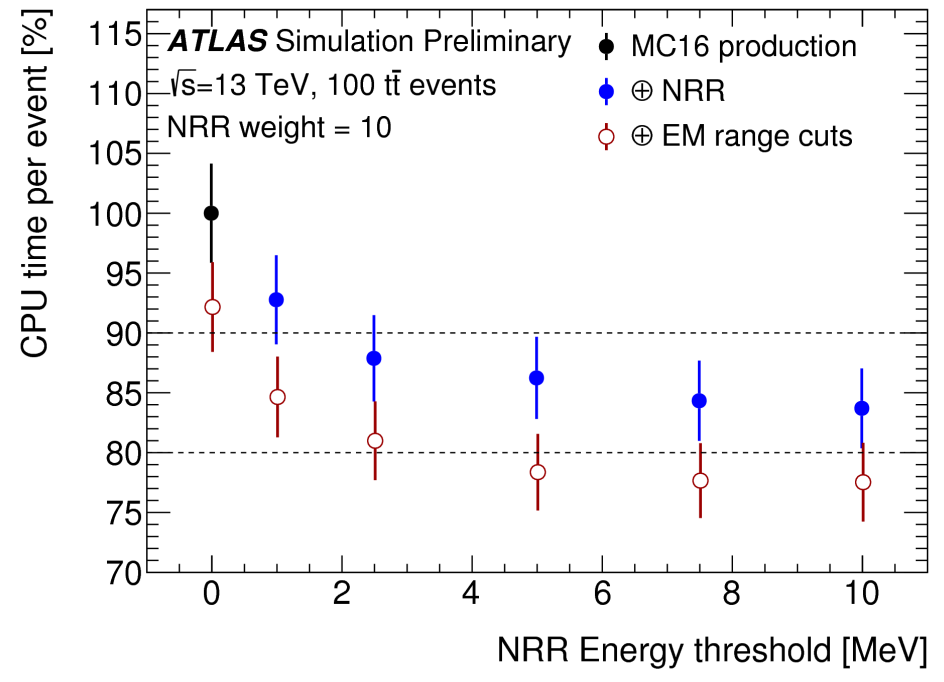

CPU time/evt. for various thresholds of NRR

The black circle represents the average for the default setup. The blue circle shows the change as the NRR threshold is increased, and the red circles the change when the EM cuts in range are modified
Other ATLAS activities and needs in the area of physics models for simulation

- Using G4 hadronic interaction models directly within its fast tracker simulation (FATRAS)

- Interested in physics models of hadronic interactions for b-hadrons for quasi-stable particle simulation.

- Studies of the impact of the choice of physics list on jet systematics is underway

See ATLAS' G4Performance optimization plots here

(The CMS experiment also implemented NRR, achieving a $25-30 \%$ reduction in CPU execution time.) 


\section{Detector simulation physics: $\mathrm{LHCb}$}

Experiment at the LHC primarily designed to search for indirect evidence of New Physics in CP violation and rare decays of beauty and charm hadrons

- Hadronic cross sections and models important for a spectrometer with particle ID

- Particle multiplicity and kinematics

- Particle/anti-particle asymmetry in the tracker

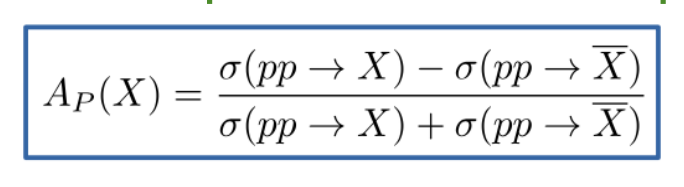
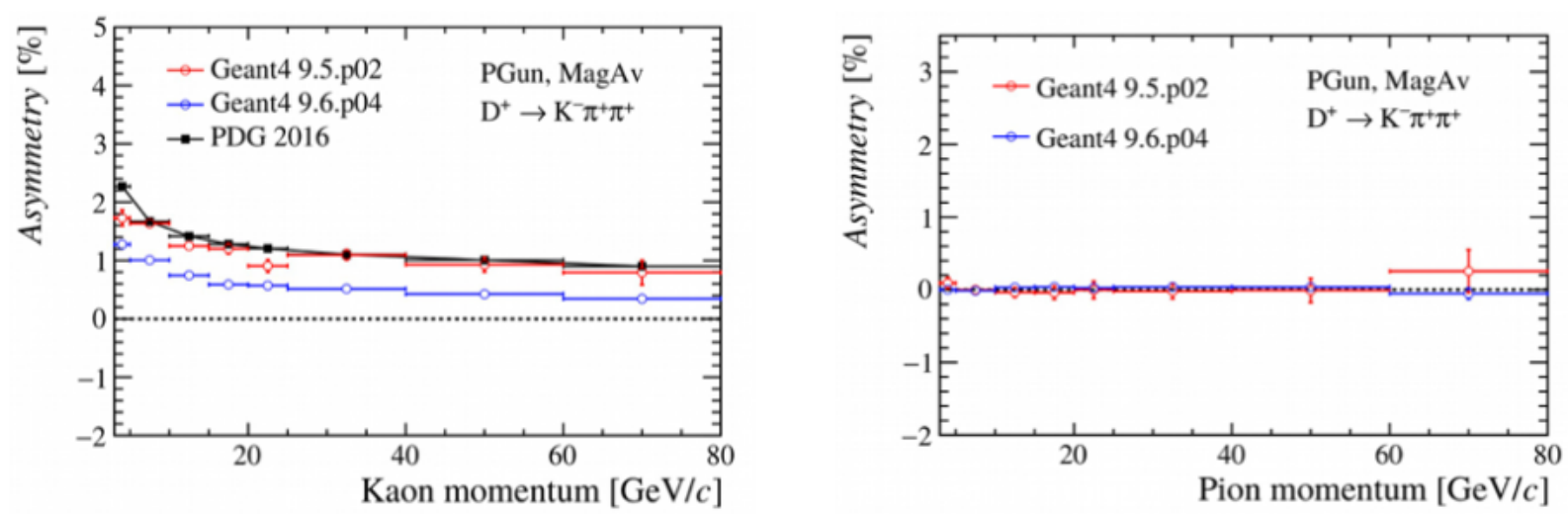

Geant4 v96r4p0g1 (contains LHCb additions)

FTFP_BERT used for $\mathrm{K}$ and $\pi$ studies

Pion asymmetry agrees with expectations, Kaon asymmetry is low (fixed in G4 10.3.p03) Proton asymmetry (not shown) well described with G4 9.5p02 - needs update 


\section{Detector simulation physics: LHCb}

- Vertex LOcator (VELO) is being upgraded for the LHC Run3 for increased precision

- EM physics modeling, including multiple scattering, is essential for simulation of tracker

- Impact parameter (IP), primary vertex (PV) resolution

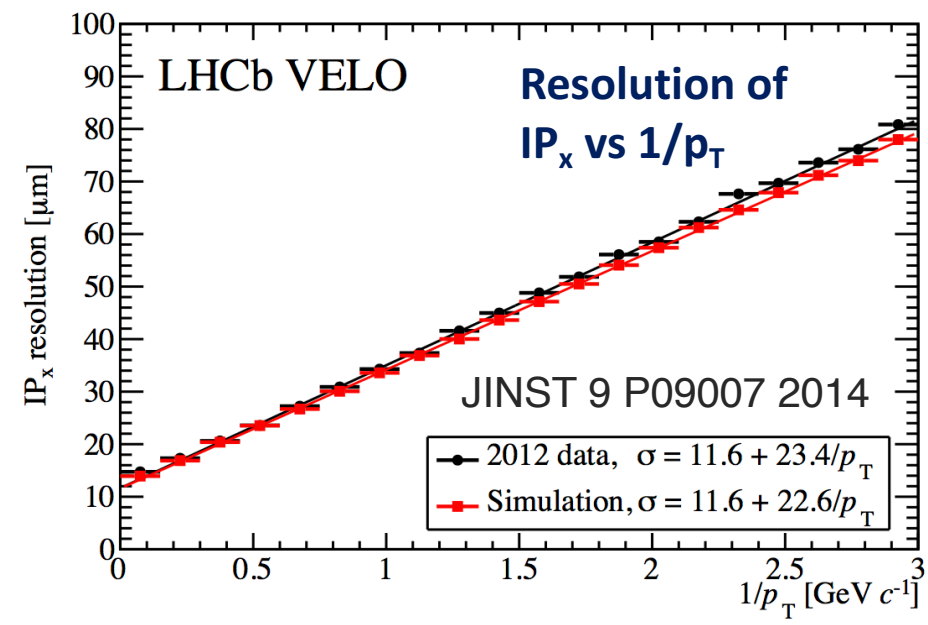

2012 simulation

Geant 4.9.5.p02

FTFP_BERT

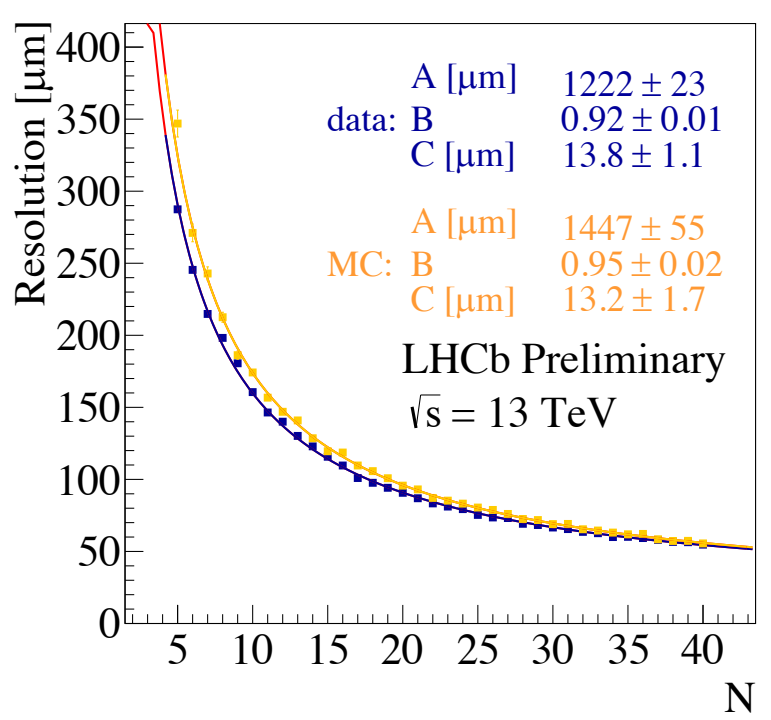

VELO simulation in good agreement with data - IP resolution excellent, PV x, y resolution within stats. (not shown), PV z resolution lower than data 


\section{Detector simulation physics: $\mathrm{LHCb}$}

- LHCb's RICH detector needs reliable modeling of Cerenkov processes

- Qualitative agreement between simulation and data for particle ID quantities

- Efforts for detailed MC-to-data comparisons have started

- LHCb's muon detector needs modeling of backgrounds from low energy neutrons, protons, pions, kaons, muons

- Full geometry and low particle production thresholds in G4 is CPU intensive

- Solution: parametrization of hits in full geometry, low threshold G4 simulation

- For run 2 conditions, the MC rate as compared to data is $2 x$ lower in the inner regions and 10$20 \%$ lower in the outer regions

Detailed simulation of $\mathrm{RICH}$, including background effects, recently optimized for speed Low energy background parametrization in muon detectors being updated (Using best physics models available in Geant4) 


\section{Detector simulation physics: ALICE}

Heavy-ion detector at the $\mathrm{LHC}(\mathrm{Pb}-\mathrm{Pb}, \mathrm{p}-\mathrm{Pb})$ to study quark-gluon plasma, using system of 17 sub-detectors for complete particle identification

- Light nuclei measurements need FTFP_INCLXX_EMV physics list

- Dominant background produced by spallation reactions in interactions between primary particles and the detector material or the beam pipe

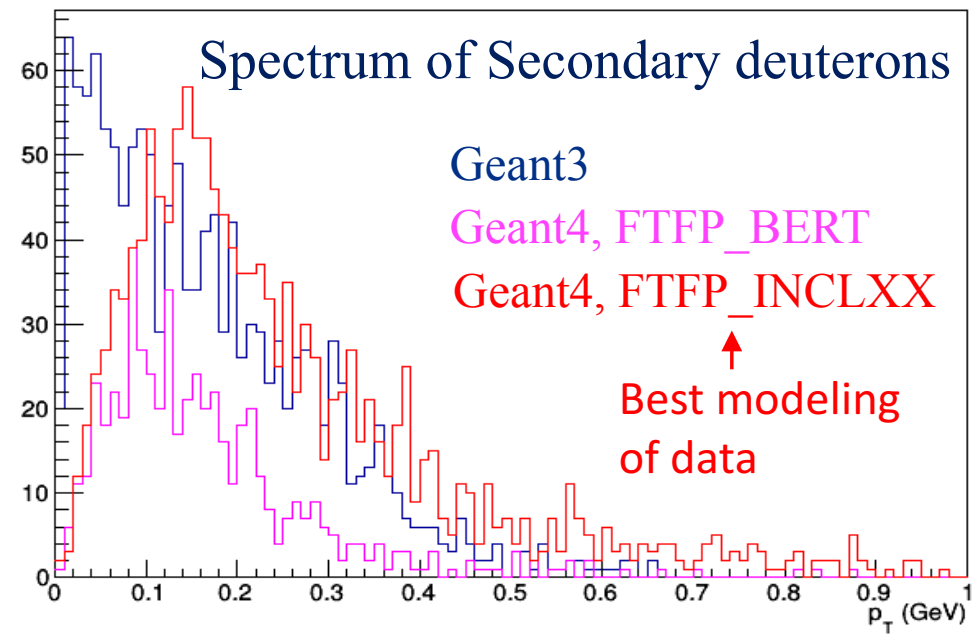

- Bkgd. estimation method depends on MC modeling of secondary light nucleon

- The INCL++ model is the best because it has coalescence implemented

ALICE using G4 10.1.p03 with

FTFP_INCLXX_EMV ( 2.1 slower!)

Preference to have light nucleons correctly implemented in FTFP_BERT_EMV instead 


\section{Detector simulation physics: Belle II}

Searches for new physics at the SuperKEK B factory $\mathrm{e}^{+} \mathrm{e}^{-}$collider (4 onto $7 \mathrm{GeV}$ )

- Belle Il uses G4 10.1 with the FTFP_BERT physics list (+ optical physics)

- Plan to migrate to G4 10.5 and develop a dedicated physics list

- Improve hadronic contribution to shower shapes while reducing CPU consumption

- Main features of dedicated physics list are:

- Use of EM option 1 (EMV extension), which cuts out low energy e- produced by photons therefore improving CPU performance without sacrificing physics too much

- Give more weight to Bertini Cascade Model for more accuracy at low energies (broader showers) and higher speed

- Add optical photons option

- Add high performance neutron option for background studies near the beamline

Validation will compare different options for speed as well as physics accuracy for shower shapes, e/p, visible energy - developer (D. Wright) is a G4 member/expert 


\section{Summary and outlook}

HL-LHC and B factory experiments in the 2020's require higher physics simulation accuracy and lower execution times, simultaneously

- ALICE, ATLAS, CMS, and LHCb report some disagreements of Geant4 predictions for shower energy fluctuations and lateral shapes, particularly in the low energy range

- Best models for detailed shower description come at a high time performance cost

- LHCb reported a low kaon cross section asymmetry in G4 prompted a fix in v10.3.p03

- Belle II is developing a dedicated physics list to increase the contribution of hadronic models (Bertini) and achieve broader showers at low energies

- LHCb needs reliable modeling of Cerenkov processes

- ALICE needs correct light nucleon interactions ported to the FTFP_BERT physics list

The experiments and G4 collaborate effectively in the area of physics validation

Recent G4 physics improvements and future plans are described in the HSF Detector Simulation CWP and reported by the G4 Collaboration at the 2018 HSF Workshop in Napoli. (Also see backup slides.) 


\section{Acknowledgements}

This presentation was composed of contributions from the ALICE, ATLAS, CMS, $\mathrm{LHCb}$, and Belle II experiments, as well as from Geant4 members

It should represent the physics simulation status and needs of the experiments as described by their detector simulation conveners, who provided the material shown 


\section{Backup slides}




\section{Recent G4 physics improvements and plans for the future}

Described in the HSF Detector Simulation CWP and reported by the G4 Collaboration at the 2018 HSF Workshop in Napoli

- Electromagnetic physics

- Developed a Goudsmit-Saunderson "theory-based" model for $\mathrm{e}^{+}$and $\mathrm{e}^{-}$, which outperforms the current Geant4 models without a CPU penalty. (Available since G4 10.2.)

- Introduced of Landau-Pomeranchuk-Migdal (LPM) effect in bremsstrahlung process which plays a role at high energies. (Improved in G4 10.3.)

- Added a relativistic pair-production model, essential for Future Circular Collider (FCC) studies. (Improved in G4 10.4.)

- Developed elastic scattering models with nuclear form factors for finite nuclear size effects at extreme high energies, important for FCC studies. (Available since G4 10.3.)

- All EM physics models extended to $100 \mathrm{TeV}$ in Geant4 10.3

- Future efforts will focus on theoretical reviews of all EM physics models, as well as the development of fluctuation models based on theory, not parametrization 


\section{Recent G4 physics improvements and plans for the future}

Described in the HSF Detector Simulation CWP and reported by the G4 Collaboration at the 2018 HSF Workshop in Napoli

- Hadronic physics

- High precision (HP) neutron library now includes revised neutron capture cross-sections and final state generator which should improve lateral shower shapes and shower time structure (Available since G4 10.0 in all non HP physics lists.)

- The Fritiof (FTF) model was recently improved and re-tuned. (Starting in G4 10.5.)

- Future efforts will focus on a thorough review of the hadronics sector:

- Further improvement of the QGS model, which offers better confidence than FTF at high energies up to a few $\mathrm{TeV}$

- Implementation of the EPOS hadronic generator for very high energy and cosmic ray physics

- Further development of the INCL++ model

- In physics lists, combination of Bertini, Binary and INCL++ for optimization of accuracy and speed

- Review cross-sections for hadron-nucleus, nucleus-nucleus and anti-nucleus nucleus reactions 